\title{
Localization of two interacting electrons in quantum dot arrays driven by an ac field
}

\author{
C. E. Creffield \\ Dipto di Fisica, Università di Roma “La Sapienza," Piazzale Aldo Moro 2, I-00185 Rome, Italy \\ G. Platero \\ Instituto de Ciencia de Materiales (CSIC), Cantoblanco, E-28049 Madrid, Spain
}

(Received 26 August 2003; published 19 April 2004)

\begin{abstract}
We investigate the dynamics of two interacting electrons moving in a one-dimensional array of quantum dots under the influence of an ac field. We show that the system exhibits two distinct regimes of behavior, depending on the ratio of the strength of the driving field to the interelectron Coulomb repulsion. When the ac-field dominates, an effect termed coherent destruction of tunneling occurs at certain frequencies, in which transport along the array is suppressed. In the other, weak-driving, regime we find the surprising result that the two electrons can bind into a single composite particle-despite the strong Coulomb repulsion between themwhich can then be controlled by the ac field in an analogous way. We show how calculation of the Floquet quasienergies of the system explains these results, and thus how ac fields can be used to control the localization of interacting electron systems.
\end{abstract}

DOI: 10.1103/PhysRevB.69.165312

PACS number(s): 73.23.- b, 72.15.Rn, 03.67.Mn, 42.50.Hz

\section{INTRODUCTION}

Recent years have seen considerable progress in the theoretical and experimental investigation of electron dynamics in mesoscopic systems. Preservation of the quantum coherence of the electronic states can give rise to unusual transport phenomena, one of the most notable of which is termed "coherent destruction of tunneling" 1,2 (CDT), or alternatively "dynamical localization." This occurs when the tunneling of a particle between potential minima is suppressed, thus localizing the particle, under the influence of an oscillatory electric field. The degree of suppression depends sensitively on the parameters of the driving field, thereby giving the attractive possibility of coherently manipulating electronic states in semiconductor nanostructures by means of laser pulses or oscillating gate potentials.

The presence of CDT was first recognized in quantum well systems ${ }^{1,2}$ and superlattices, ${ }^{3-5}$ in which the effects of interelectron interactions are negligible due to the high concentration of carriers. More recent work ${ }^{6-9}$ has focused on quantum dot (QD) systems, which give the ability to study a small number of electrons in a highly controllable environment. In such nanostructures, however, the low electron density means that correlations produced by the Coulomb interaction cannot be neglected, and indeed can have extremely significant effects. ${ }^{10,11}$ Numerical investigations of a system of two electrons in a double QD have revealed novel forms of CDT, resulting from the interelectron Coulomb interaction, which have been successfully analyzed by means of the Floquet approach. ${ }^{10}$ Here we extend and generalize these results by investigating the case of two electrons confined to a one-dimensional array of QDs, and make a detailed study of the interplay between the driving field and the Coulomb interaction on coherent transport. Although interesting in its own right, such understanding is also of practical importance to the field of quantum computation, ${ }^{12}$ as this type of structure provides a promising method of implementing scalable arrays of quantum bits. ${ }^{13}$
In this investigation we first demonstrate that the Coulomb interaction produces two regimes of behavior, namely, when the driving is stronger or weaker than the Coulomb interaction. This was seen previously in the double QD system, ${ }^{10}$ and we show that it is a generic feature, common to all sizes of array. In the strong-driving regime CDT occurs when the field frequency is in resonance with the Coulomb energy at certain field strengths which can be predicted by Floquet theory. ${ }^{10}$ In the weak-driving regime, however, no resonance effects arise, and initial states in which a QD is doubly occupied show a surprising behavior ${ }^{9,14,15}$ in that despite the strong Coulomb repulsion between them, the two electrons can bind together to form a single composite particle of charge $2 e$. The localization and tunneling dynamics of this two-electron state can then be controlled by suitably choosing the parameters of the ac field, in a similar way to the control of a single particle.

\section{MODEL AND METHODS}

We consider a system of two electrons confined to a onedimensional array of QD's, with a time-dependent electric field $F(t)$ applied parallel to the array. Such an array can be produced experimentally by, for example, gating a twodimensional electron gas confined in a semiconductor heterostructure. ${ }^{13,16}$ We model the QD array as a single-band tight-binding model of Hubbard type. For the double QD case, explicit calculation has shown that such an effective model is able to reproduce well the behavior of a more realistic model, ${ }^{10}$ and accordingly for an array of $N$ QD's we use the effective lattice Hamiltonian

$$
H=-\tilde{t} \sum_{\langle i, j\rangle, \sigma}^{N}\left[c_{i \sigma}^{\dagger} c_{j \sigma}+\text { H.c. }\right]+\sum_{j=1}^{N}\left[\widetilde{U} n_{j \uparrow} n_{j \downarrow}+e a F(t) j n_{j}\right] .
$$

Here $\widetilde{U}$ is the Hubbard- $U$ term giving the energy cost for double occupation of a $\mathrm{QD}$, and $\tilde{t}$ is the hopping parameter 
between adjacent QD's. In experiment $\tilde{t}$ can be set by regulating the height of the interdot tunneling barriers, ${ }^{16}$ and $\widetilde{U}$ by adjusting the size of the QD's, since $\widetilde{U} \sim 1 / L$ for a QD of size $L$. We shall measure all energies in units of $\tilde{t}$, and set $\hbar=1$. The operators $c_{j \sigma} / c_{j \sigma}^{\dagger}$ are the electronic annihilation/ creation operators, $n_{j \sigma}$ is the usual number operator, and $n_{j}$ $=n_{j \uparrow}+n_{j \downarrow}$. The interdot spacing is denoted by $a$, and for simplicity we shall describe the time-dependent electric field in terms of the potential difference between neighboring sites, $E(t)=e a F(t)$, which we parametrize as

$$
E(t)=E \sin \omega t, \quad T=2 \pi / \omega .
$$

Experimental investigations of semiconductor QD's have revealed that typically the spin relaxation time within these structures is very long ${ }^{17}$ and accordingly we do not include any spin-flip terms in the Hamiltonian (1). As a result, the singlet and triplet subspaces of the model are completely decoupled. In this work we consider just the singlet subspace, as in the triplet subspace the Pauli principle forbids double occupation of a QD, and the Coulomb interaction described by the Hubbard- $U$ term is consequently irrelevant. Suitable basis states for the spatial component of the twoelectron wave function can be obtained by taking symmetric combinations of single-particle states defined on the lattice sites $m$ and $n$ :

$$
\begin{gathered}
|m, n\rangle=\frac{\phi_{m}\left(r_{1}\right) \phi_{n}\left(r_{2}\right)+\phi_{n}\left(r_{1}\right) \phi_{m}\left(r_{2}\right)}{\sqrt{2}}, m \neq n ; \\
|m, n\rangle=\phi_{m}\left(r_{1}\right) \phi_{n}\left(r_{2}\right), \quad m=n,
\end{gathered}
$$

where $r_{1}, r_{2}$ are the coordinates of the two electrons. It can be clearly seen that the basis consists of $N(N-1) / 2$ entangled states [Eq. (3)] in which the two electrons occupy different QD's, with the remaining $N$ factorizable states [Eq. (4)] being those in which a QD is doubly occupied. For strong values of the Coulomb interaction the energies of these two types of states will fall into two bands, separated approximately by the Hubbard gap $\widetilde{U}$.

Our numerical investigation consists of initializing the system in a given state, and propagating it forward in time under the action of the time-dependent Hamiltonian (1) over many (typically between ten and one hundred) cycles of the driving field. This can be done efficiently by a fourth-order Runge-Kutta method. To study its time evolution quantitatively, we measure the projection of the two-electron wave function onto the basis states

$$
P_{m n}(t)=|\langle\Psi(t) \mid m, n\rangle|^{2}
$$

together with the distribution of electronic charge within the array, and at all times we check the normalization of the wave function to verify that unitarity is adequately preserved by the numerical procedure.

As the Hamiltonian (1) is periodic in time, $H(t)=H(t$ $+T)$, the Floquet theorem allows us to write solutions of the time-dependent Schrödinger equation as $\left|\Psi_{j}(t)\right\rangle=\exp$ $\left[-i \epsilon_{j} t\right]\left|\phi_{j}(t)\right\rangle$, where $\left|\phi_{j}(t)\right\rangle$ are termed Floquet states and $\boldsymbol{\epsilon}_{j}$ are the Floquet quasienergies. The Floquet states have the same periodicity as the Hamiltonian, and are eigenvectors of the unitary time-evolution operator for one period of the driving:

$$
U\left(t_{0}+T, t_{0}\right)=\mathcal{T} \exp \left[-i \int_{t_{0}}^{t_{0}+T} H\left(t^{\prime}\right) d t^{\prime}\right],
$$

where $\mathcal{T}$ signifies time ordering. The corresponding eigenvalues of this operator are related to the quasienergies ${ }^{18}$ via $\lambda_{j}$ $=\exp \left[-i \epsilon_{j} T\right]$. As the Floquet states provide a complete basis, the two-electron wave function can be expanded as

$$
|\Psi(t)\rangle=\sum \alpha_{j} \exp \left[-i \epsilon_{j} t\right]\left|\phi_{j}(t)\right\rangle .
$$

This expansion is directly analogous to the expansion of a wave function in energy eigenstates in the case of a timeindependent Hamiltonian, and indeed in the adiabatic limit $T \rightarrow \infty$ the quasienergies evolve into eigenenergies, and the Floquet states to eigenstates. From Eq. (7) it is clear that the long time scale behavior of the system is dictated essentially by just the quasienergies, since the Floquet states are periodic with period $T$, and also generally have a weak time dependence. Tunneling between two states is suppressed ${ }^{19}$ when their quasienergies approach each other as an external parameter (such as the electric field) is varied. Thus as well as being directly observable in the behavior of the electronic wave function, CDT may also be detected in the system's quasienergy spectrum by the presence of exact or avoided crossings. We shall show in the following section how these crossings in the exact quasienergies, obtained from the numerical diagonalization of the time-evolution operator (6), correspond to the suppression of the dynamics of the electrons, and how analytic approximations to the quasienergies allow the parameters at which this occur to be predicted with high accuracy.

\section{RESULTS}

In Fig. 1 we show the exact quasienergy spectra with parameters $\omega=2$ and $\widetilde{U}=16$, for two extreme cases of array size: a two-site system (i.e., a double QD) and a large system of $N=16$ sites. To assist the comparison between the two systems, the quasienergies have been classified into three categories according to how the electrons are distributed within the QD array in the corresponding Floquet state. To determine this we first class the basis states as (i) states in which both electrons occupy the same QD ("doubly occupied states"), (ii) states in which the electrons occupy neighboring QD's ("neighbor states"), and (iii) states in which the electrons are more widely separated. We then assign each Floquet state to the class onto which it projects the most, according to the magnitude of the overlap integrals $\left|\left\langle\phi_{j}(0) \mid m, n\right\rangle\right|^{2}$.

Some similarities between the two spectra in Fig. 1 are immediately clear. In the two-site case a pair of quasienergies make a sequence of exact crossings as $E$ is increased from zero up to $\widetilde{U}$, while the remaining quasienergy stays isolated from them. As the two intercrossing states evolve 


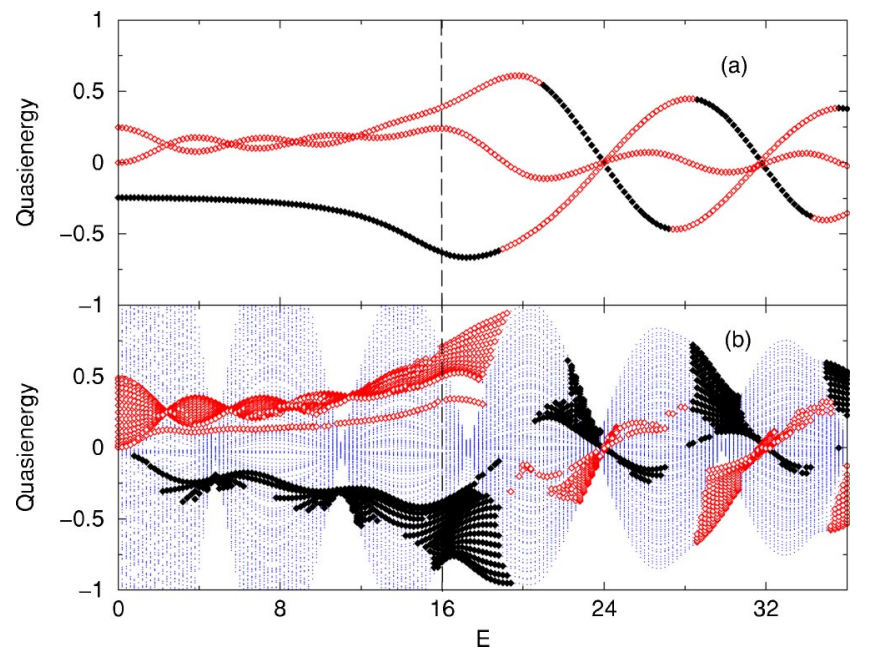

FIG. 1. (Color online) Quasienergy spectrum for (a) a two-site system, and (b) a 16-site system, for $\widetilde{U}=16$ and $\omega=2$. Symbols indicate the characteristic of the corresponding Floquet state: hollow diamonds show doubly occupied states, solid diamonds show neighbor states, and points show states with wide separation between electrons. The widely separated states (points) exhibit a series of miniband collapses in excellent agreement with Eq. (8) over the whole range of $E$. For weak driving the doubly occupied states show a similar set of collapses but with half the period. The vertical dashed line marks the boundary between the weak- and strongdriving regimes.

from the excited eigenstates of the static Hamiltonian, they consequently project mainly onto the doubly occupied states. The remaining, isolated, Floquet state evolves from the system's ground state and therefore has the character of a neighbor state.

In the 16-site system we can see a similar, but richer, behavior. Instead of just two intercrossing states in the weakfield regime, the set of doubly occupied states (4) form a miniband, similar to that observed for noninteracting electrons in Refs. 4,20. In analogy to the crossings seen in the two-site system, this miniband exhibits a sequence of "band collapses" at which the quasienergies become degenerate. These collapses occur at the same values of $E$ at which the crossings occur in the two-site case. This behavior is seen more clearly in Fig. 2(a) for a nine-site system. Here seven of the doubly occupied quasienergies make up a miniband and exhibit a set of band collapses as $E$ is increased, while the two remaining states, which physically correspond to the two edge states, are almost degenerate and show little dependence on $E$.

For non interacting particles, Holthaus ${ }^{20}$ obtained an approximate expression for the quasienergy spectrum:

$$
\epsilon_{j}=\frac{\Delta}{2} J_{0}(E / \omega) \cos a k_{j},
$$

where $J_{0}$ is the zeroth Bessel function of the first kind, $\Delta$ is the width of the miniband, and $k_{j}$ are the permitted lattice momenta (assuming periodic boundary conditions). In the system we consider, the Coulomb interaction only operates if both electrons occupy the same QD. Accordingly, if the elec-

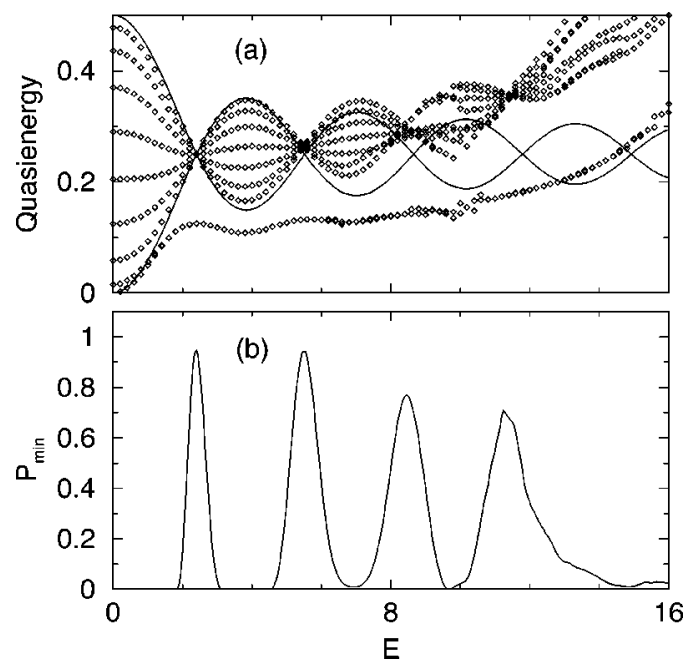

FIG. 2. Weak-field regime for a nine-site system, $\widetilde{U}=16$ and $\omega=2$. (a) Seven quasienergies form a miniband, while the two almost degenerate edge states remain isolated from the rest. The solid lines are the approximate solution $\pm(\Delta / 2) J_{0}(2 E / \omega)$, where $\Delta$ is the width of the miniband. As $E$ approaches $\widetilde{U}$ the miniband structure progressively breaks down. (b) Minimum value of $P_{55}(t)$ for this system, initialized in state $|5,5\rangle$. At the points of quasienergy degeneracy (miniband collapse) CDT occurs.

trons are distant from each other they behave effectively as free particles, and in Fig. 1(b) we can see that the Floquet states in which the two electrons are widely separated (plotted with points) form a miniband of exactly the type given by Eq. (8). Clearly, these states cannot arise in the two-site system, as it is impossible for the two electrons to be further apart than a single lattice spacing.

Surprisingly, Holthaus' result for noninteracting particles can also be applied to understand the miniband behavior of the doubly occupied states, in which the Coulomb interaction is maximized. In this case, as the miniband is composed of Floquet states that project onto doubly occupied states, it is plausible to visualize the localized two-electron state as tunneling from site to site as a single object. Since this object has charge $2 e$ the period of the miniband is consequently halved, taking the form $\epsilon_{j}=(\Delta / 2) J_{0}(2 E / \omega) \cos a k_{j}$. In Fig. 2(a) we plot the envelope of this miniband, and see that for low values of $E$ the agreement with the exact results is excellent. As the value of $E$ increases, however, the doubly occupied Floquet states begin to mix and interact with the other states, and the miniband structure starts to break down. When $E$ exceeds the size of the Hubbard gap the structure is completely lost, and the system enters the strong-driving regime.

To illustrate the physical significance of the miniband collapses, we show in Fig. 2(b) the minimum value of the probability $P_{55}(t)$ attained by the system during ten periods of the driving, having been initialized in the state $|5,5\rangle$ - that is, with both electrons in the central QD. It can readily be seen that at the points of miniband collapse the system remains frozen in its initial state, thereby manifesting CDT, and producing sharp peaks in $P_{\min }$ centered on the points of quasienergy degeneracy. This demonstrates that interpreting the two- 


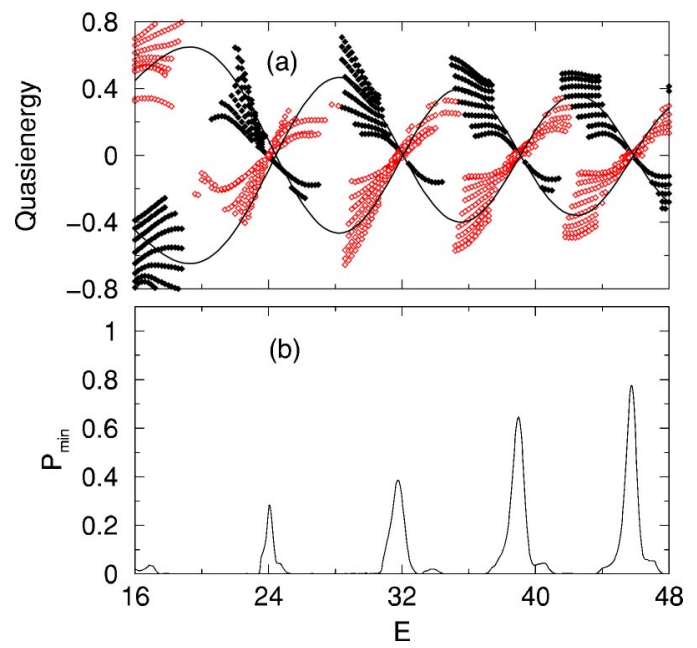

FIG. 3. (Color online) Strong-field regime for the nine-site system. (a) Crossings occur between Floquet states of doubly occupied type (hollow symbols) and those of neighbor type (solid symbols). The lines are the perturbative solution $\pm 2 J_{8}(E / \omega)$. (b) Minimum value of $P_{55}(t)$ for this system, initialized in state $|5,5\rangle$. CDT again occurs when the quasienergies cross.

electron state as a single composite particle, as suggested by the form of the Floquet spectrum, indeed provides the correct description of the system's dynamics.

To complete the comparison of the two cases in Fig. 1, we now consider the strong-driving regime $(E>\widetilde{U})$. Again, clear similarities between the two quasienergy spectra are evident. In the case of the two-site system, a pair of the quasienergies make a set of close approaches while the third oscillates weakly around zero. Detailed examination of the close approaches (Ref. 10) reveals that they are in fact avoided crossings, and a perturbative treatment of this system performed in that work yields the approximate solution:

$$
\epsilon_{ \pm}= \pm 2 J_{n}(E / \omega)
$$

where $n \omega=\tilde{U}$. When this resonance condition between $\tilde{U}$ and the driving frequency is not satisfied, the quasienergies in this regime show little structure, and CDT is in general not produced. In Fig. 1(b) it is clear that a generalization of this phenomenon occurs, and a similar set of close approaches between neighbor and doubly occupied Floquet states occurs. In Fig. 3(a) we plot the quasienergies of the neighbor and doubly occupied Floquet states for a nine-site array in this regime, and find that the locations of the quasienergy degeneracies are again given accurately by the perturbative result [Eq. (9)], namely at the roots of $J_{8}(E / \omega)$. It can also be seen that the agreement between the perturbative approximation and the exact result improves as $E$ increases. This is to be expected, as the "perturbation" used in the derivation of Eq. (9) consists of the tunneling component of the Hamiltonian, and thus the result becomes exact in the limit of large $E$ when the tunneling is negligible in comparison with the ac driving.

In Fig. 3(b) we confirm that the structure seen in the quasienergy spectrum indeed corresponds to a modification of the system's dynamics. As before, the system is initialized

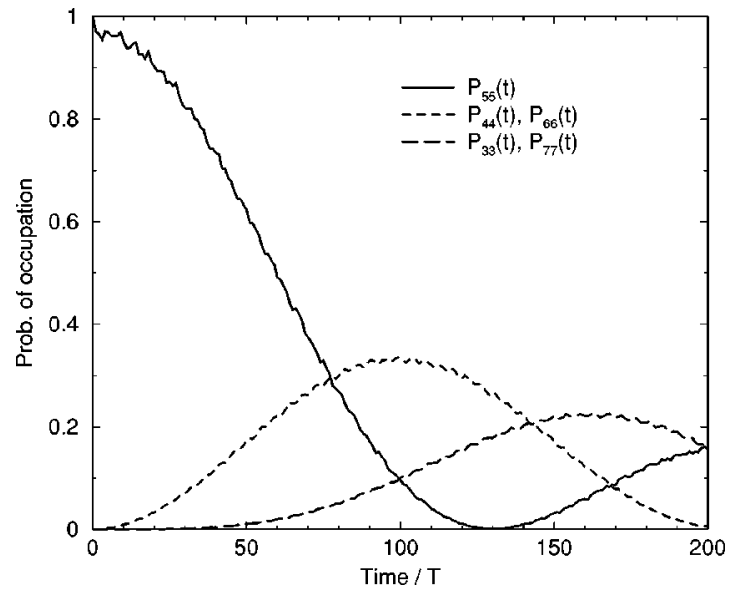

FIG. 4. Time-dependent projection of the two-electron state onto the basis functions [see Eq. (5)] with the system initialized in state $|5,5\rangle$. Only the projections onto the doubly occupied basis states are shown, as all the others are negligible in size. Field parameters: $E=2.35, \omega=2$. The differences between $P_{44}$ and $P_{66}$, and between $P_{33}$ and $P_{77}$, are too small to be seen here.

in state $|5,5\rangle$, and integrated over ten cycles of the driving field. The sharp peaks again visible in $P_{\min }$ are located at the points of quasienergy degeneracy, indicating that CDT occurs as expected. Thus in both weak- and strong-driving regimes, Floquet analysis can be used to predict the field parameters at which CDT will occur in a driven QD array with high accuracy.

We now examine the dynamics of the system in the weakdriving regime in more detail. If the system is initialized in a state in which both electrons occupy the same QD, one would expect that the Coulomb repulsion would act to repel the two electrons away from each other. We have already seen, however, that this does not occur, and that in the presence of the ac field the two electrons in fact bind together and propagate as a single composite particle. ${ }^{14,15}$ Although surprising, the ability of an ac field to convert a repulsive interaction into a time-averaged attraction forms the basis of an increasing number of applications, ranging from the wellknown Paul trap ${ }^{21}$ to a recent proposal to bind atomic nuclei together $^{22}$ with laser fields. We show in Fig. 4 the explicit time dependence of the dominant components of the wave function of a nine-site system, initialized with both electrons in the central QD. The strength of the electric field has been set to $E=2.35$, close to the point of the first miniband collapse at $E \simeq 2.4048$, and thus the effective tunneling is heavily suppressed but nonzero. It can be seen that over the first hundred periods of the driving the initial state $|5,5\rangle$ evolves chiefly into a superposition of the states $|4,4\rangle$ and $|6,6\rangle$, by diffusing symmetrically ${ }^{23}$ to the neighboring QD's, while retaining the two-particle superposition. This is confirmed by the fact that throughout the time evolution, the total projection of the wave function onto the doubly occupied states never takes a value of less than 0.955 , indicating that the role of the other states is merely to act as short-lived intermediates in the diffusion process.

Figure 5 vividly demonstrates how the initially localized two-electron state on site 5 splits smoothly into two pulses 


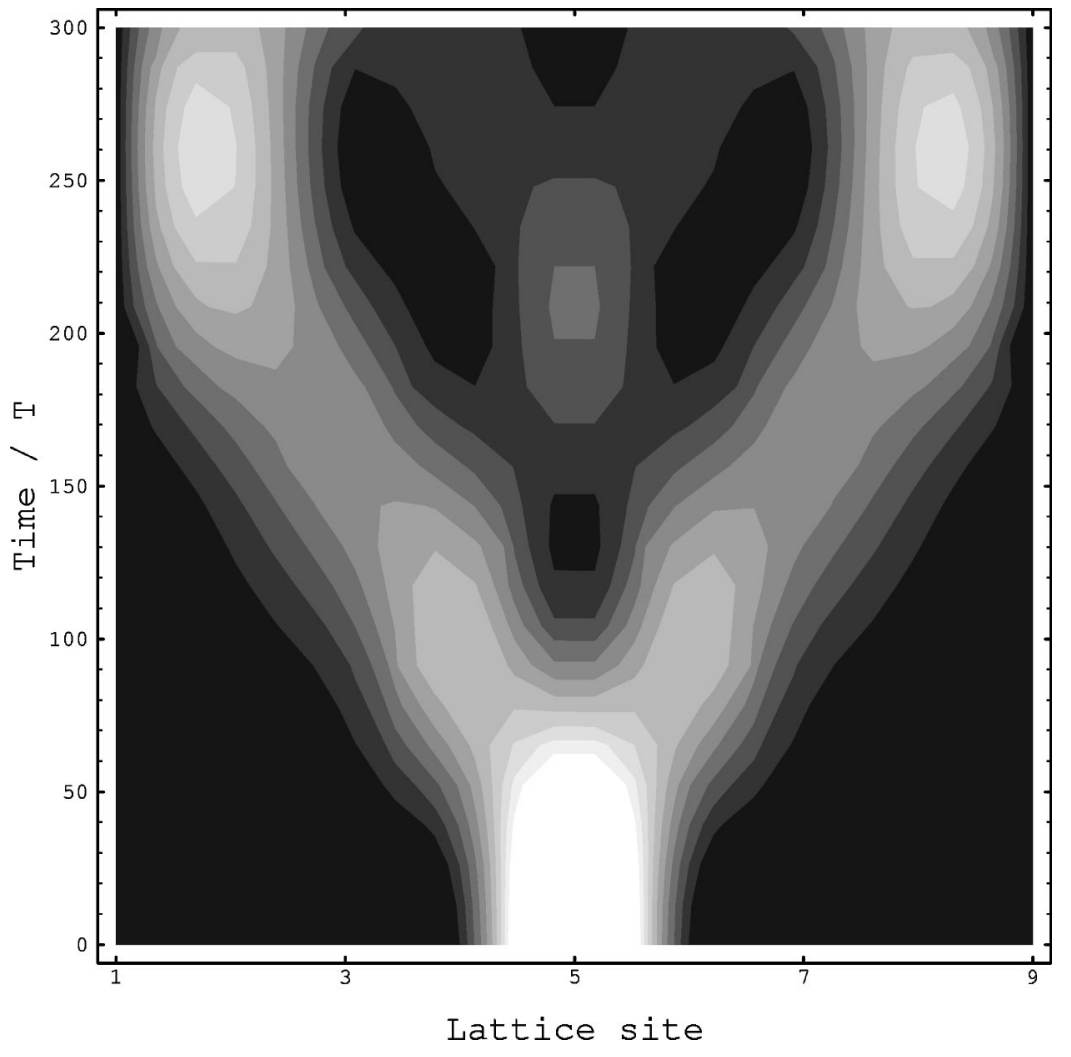

FIG. 5. Time-dependent charge-density $|\Psi(t)|^{2}$ for a nine-site lattice, with the initial condition of both particles on site 5 (the central site). White/black signifies high/low values of density. Field parameters are $E=2.35, \omega=2$. moving to the left and right at an almost constant velocity. Although reminiscent of soliton dynamics, the lack of any dispersive effects to provide "refocusing" of the wave front means that as the pulses traverse the system they do smear out to an extent. A result of this, for example, is that the peak in $P_{33}$ does not rise to the same height as $P_{44}$ in Fig. 4 due to blurring of the pulse shape. Even so, the definition of the charge pulses is sufficiently crisp to suggest the possibility of using an array of QD's as a type of coherent electron turnstile. ${ }^{24}$ In such a device the ac field is used to control the rate at which the charge pulses propagate, so that in a given interval of time a specified quantity of charge is transferred along the array. The well-defined trajectories of the current pulses visible in Fig. 5 indicate that the velocity of the pulse can be unambiguously measured, and in Fig. 6 we give a plot of this quantity, obtained as the reciprocal of the time required for the initial QD to become empty. Since the velocity of the pulse is directly related to the magnitude of the interdot tunneling, the velocity shows the same Bessel function dependence as the quasienergies, and vanishes at field parameters such that $J_{0}(2 E / \omega)=0$, at which the tunneling is completely suppressed. This clearly shows how the ac field can be used as a control parameter to regulate the speed of propagation of the pulse by means of CDT.

It is interesting to note that this arrangement generates an unusual current of entangled, correlated electrons. As the initial state propagates symmetrically to the left and right of the QD array, there is an equal probability of finding an individual electron on either side of the central QD, but due to the combined effect of the ac field and the Coulomb interaction there is a very high chance that the other electron will also be occupying the same QD. Recently Saraga and Loss ${ }^{25}$ have proposed using an array of three QD's to create currents of entangled electrons for use in quantum computing and communication applications. We note that their setup contains many similarities with the system we study here: a pair of electrons is injected into the central QD, which then emits electron pulses to its neighbors. The major difference is that in Ref. 25 the current is composed of single electrons (in a superposition of up and down spin states), whereas in our case the current is carried by a two-electron bound state. In

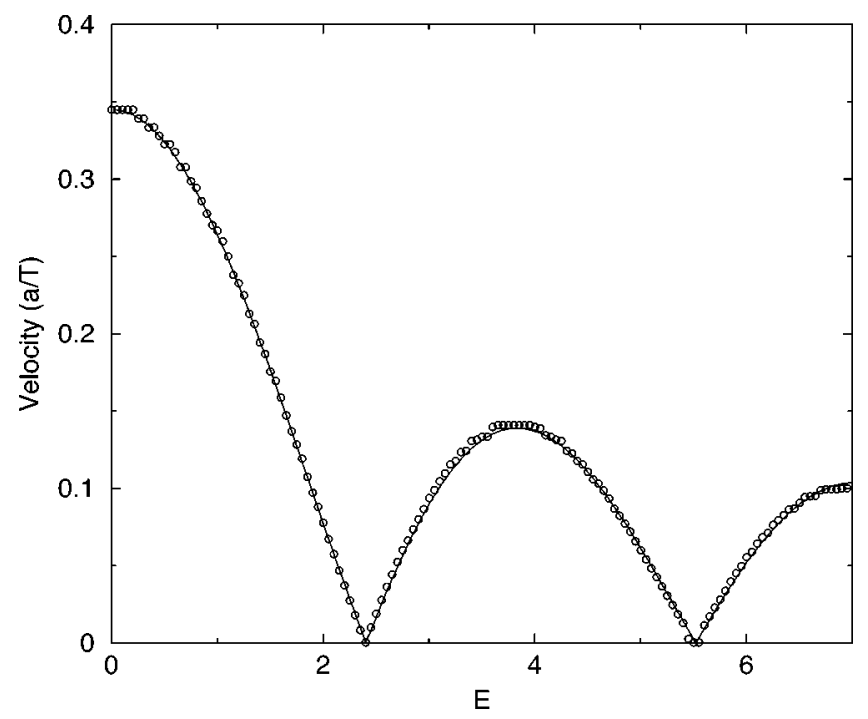

FIG. 6. Circles indicate the velocity of the charge pulse in the nine-site system as it propagates through the array from the initial state $|5,5\rangle$. The solid line is $v_{0}\left|J_{0}(2 E / \omega)\right|$, where $v_{0}$ is a fitting parameter. 
this sense the driven QD array that we study can be regarded as an electronic analog of an optical beam splitter, ${ }^{26}$ which divides the initial state into two spatially separated and entangled electron pairs moving at a velocity controlled by the ac-driving field.

\section{CONCLUSIONS}

In summary, we have studied the dynamical behavior of two interacting electrons in an QD array under ac driving. We have seen that the competition between the Coulomb energy and the driving field produces a rich phenomenology, which can be described well by means of Floquet theory. Throughout the weak-field regime, the driving field and the Coulomb interaction combine in a counterintuitive way to bind the two electrons together to form a composite particle resembling an exciton. The tunneling of this particle from site to site can then be controlled by manipulating the driving field, which permits either the complete freezing of the dynamics at the points of "miniband collapse," or the fine regulation of the current of entangled electron pairs. When the field is stronger than the Coulomb interaction the character of the Floquet states abruptly changes, and a different form of CDT occurs, arising from the close approaches of states of doubly occupied type and neighbor type. This generalizes the form of CDT observed previously in a two-site system, ${ }^{10}$ and only occurs when the driving field is in resonance with the Coulomb energy (i.e., $n \omega=\widetilde{U}$ ) at the roots of $J_{n}(E / \omega)$. The delicate control over the electron dynamics afforded by these effects has many potential applications to the coherent manipulation of correlated electron states in mesoscopic systems.
Although in this work we only consider one specific set of system parameters and one driving frequency, we have found that the results are of general validity as long as $\tilde{U}>\tilde{t}$ (which is usually satisfied for QD's in the Coulomb blockade regime) and that the system is in the high-frequency regime ${ }^{2}$ $(\omega>\widetilde{t})$. A recent experimental study ${ }^{13}$ gave estimates of $\widetilde{U}$ $\simeq 3.7 \mathrm{meV}$ and $\tilde{t} \simeq 0.5 \mathrm{meV}$ for the double QD system studied there. Given that the QD separation was about $200 \mathrm{~nm}$, the required driving field to see the CDT effects we report should be of the order of $200 \mathrm{GHz}$, with field strengths of up to $10 \mathrm{kV} / \mathrm{cm}$. Such systems also possess excellent coherence properties, with decoherence times of the order of nanoseconds reported recently. ${ }^{27}$ We therefore believe that experimental observation of these effects is feasible, and could be achieved, for example, by connecting the system to external leads and measuring the current passing through the array, or more directly, by measuring the charge occupation of the individual QD's by means of quantum point contacts, as has recently been achieved in Refs. 13,28. This latter technique has the important advantages that the occupation of the QD's can be determined even if the interdot current is too small to measure by conventional means, and also should induce less decoherence than the transport form of measurement.

\section{ACKNOWLEDGMENTS}

C.E.C. acknowledges support from the physics department of the Università di Roma "La Sapienza." G.P. was supported by the Spanish DGES Grant No. MAT2002-02465 and by the EU Human Potential Program under Contract No. HPRN-CT-2000-00144.
${ }^{1}$ F. Grossmann, T. Dittrich, P. Jung, and P. Hänggi, Phys. Rev. Lett. 67, 516 (1991).

${ }^{2}$ F. Grossmann and P. Hänggi, Europhys. Lett. 18, 571 (1992).

${ }^{3}$ D.H. Dunlap and V.M. Kenkre, Phys. Rev. B 34, 3625 (1986).

${ }^{4}$ M. Holthaus, Phys. Rev. Lett. 69, 351 (1992).

${ }^{5}$ Xian-Geng Zhao, J. Phys.: Condens. Matter 6, 2751 (1994).

${ }^{6}$ P.I. Tamborenea and H. Metiu, Europhys. Lett. 53, 776 (2001).

${ }^{7}$ P. Zhang and X.-G. Zhou, Phys. Lett. A 271, 419 (2000).

${ }^{8}$ Ping Zhang, Qi-Kun Xue, Xian-Geng Zhao, and X.C. Xie, Phys. Rev. A 66, 022117 (2002).

${ }^{9}$ Emmanuel Paspalakis, Phys. Rev. B 67, 233306 (2003).

${ }^{10}$ C.E. Creffield and G. Platero, Phys. Rev. B 65, 113304 (2002).

${ }^{11}$ C.E. Creffield and G. Platero, Phys. Rev. B 66, 235303 (2002).

${ }^{12}$ D. Loss and D.P. DiVincenzo, Phys. Rev. A 57, 120 (1998).

${ }^{13}$ J.M. Elzerman, R. Hanson, J.S. Greidanus, L.H. Willems van Beveren, S. De Franceschi, L.M.K. Vandersypen, S. Tarucha, and L.P. Kouwenhoven, Phys. Rev. B 67, 161308(R) (2003).

${ }^{14}$ C.E. Creffield and G. Platero, in The Anderson Transition and its Ramifications, edited by T. Brandes and S. Kettemann (SpringerVerlag, Berlin, 2003).

${ }^{15}$ Ken-ichi Noba, Phys. Rev. B 67, 153102 (2003).

${ }^{16}$ F.R. Waugh, M.J. Berry, D.J. Mar, R.M. Westervelt, K.L. Campman, and A.C. Gossard, Phys. Rev. Lett. 75, 705 (1995).

${ }^{17}$ T. Fujisawa, D.G. Austing, Y. Tokura, Y. Hirayama, and S.
Tarucha, Phys. Rev. Lett. 88, 236802 (2002).

${ }^{18}$ The quasienergies are only defined up to an arbitrary multiple of the driving frequency, that is $\epsilon$ and $\epsilon+n \omega$ correspond to the same physical state. In this work we take the normal convention of restricting the quasienergies to the "first Brillouin zone" $(-\omega / 2 \leqslant \epsilon<\omega / 2)$ which gives a unique representation.

${ }^{19}$ M. Grifoni and P. Hänggi, Phys. Rep. 304, 229 (1998).

${ }^{20}$ M. Holthaus, Z. Phys. B: Condens. Matter 89, 251 (1992).

${ }^{21}$ W. Paul, Rev. Mod. Phys. 62, 531 (1990).

${ }^{22}$ Olga Smirnova, Michael Spanner, and Misha Ivanov, Phys. Rev. Lett. 90, 243001 (2003).

${ }^{23}$ In fact, a small asymmetry between diffusion to the left and to the right is introduced by the phase of the driving field [Eq. (2)], but after averaging over many periods of the driving this asymmetry becomes negligible.

${ }^{24}$ P.I. Tamborenea and H. Metiu, Phys. Rev. Lett. 83, 3912 (1999).

${ }^{25}$ Daniel S. Saraga and Daniel Loss, Phys. Rev. Lett. 90, 166803 (2003).

${ }^{26}$ W.D. Oliver, F. Yamaguchi, and Y. Yamamoto, Phys. Rev. Lett. 88, 037901 (2002).

${ }^{27}$ T. Hayashi, T. Fujisawa, H.D. Cheong, Y.-H. Jeong, and Y. Hirayama, Phys. Rev. Lett. 91, 226804 (2003).

${ }^{28}$ Wei Lu, Zhongqing Ji, Loren Pfeiffer, K.W. West, and A.J. Rimberg, Nature (London) 423, 422 (2003). 